\title{
Nuevos registros de flebotomíneos (Diptera: Psychodidae) en el área de influencia del río Amoyá en Chaparral, Tolima
}

\author{
María Angélica Contreras¹, Rafael José Vivero", Eduar Elías Bejarano², \\ Lina María Carrillo ${ }^{1,3}$, Iván Darío Vélez ${ }^{1}$ \\ 1 Programa de Estudio y Control de Enfermedades Tropicales, Universidad de Antioquia, Medellín, Colombia
2 Grupo de Investigaciones Biomédicas, Universidad de Sucre, Sincelejo, Colombia \\ ${ }^{3}$ Facultad de Ciencias Agrarias, Universidad de Antioquia, Medellín, Colombia
}

Introducción. En Colombia, la diversidad de flebotomíneos es alta, con 162 especies registradas que incluyen especies vectoras de Leishmania spp.

Objetivo. Identificar las especies de flebotomíneos de importancia médica dentro del área de influencia del Proyecto Hidroeléctrico del río Amoyá, Colombia.

Materiales y métodos. Los flebotomíneos fueron recolectados con trampas de luz de tipo CDC, Shannon y adhesivas, en 15 veredas del municipio de Chaparral, departamento de Tolima.

Resultados. Un total de 1.077 especímenes adultos de flebotomíneos fueron recolectados e identificados como pertenecientes a 13 especies del género Lutzomyia França, 1924 y una especie del género Warileya Hertig, 1948. Entre las especies del género Lutzomyia recolectadas, Lu. Iongiflocosa (Morales, Osorno y Muñoz, 1970), Lu. columbiana (Ristorcelli y Van Ty, 1941) y Lu. nuneztovari (Ortiz, 1954) son importantes por sus historiales epidemiológicos. Se resalta por primera vez la presencia de Lu. suapiensis (Le Pont, Torrez-Espejo y Dujardin, 1997) en Colombia y de Warileya rotundipennis (Fairchild y Herting, 1951) en el departamento de Tolima.

Conclusión. Este estudio contribuye al conocimiento de la distribución geográfica de la subfamilia Phlebotominae en Colombia y favorece localmente a la comprensión de la riqueza y taxonomía de estos insectos, para un mejor entendimiento de la transmisión de la leishmaniasis en el municipio de Chaparral.

Palabras clave: leishmaniasis, insectos vectores, Lutzomyia, biodiversidad, ecosistema andino, Colombia

New records of phlebotomine sand flies (Diptera: Psychodidae) near the Amoya River in Chaparral, Tolima

Introduction. In Colombia, the diversity of phlebotomine sand flies is high, with 162 recorded species, and which include vectors of Leishmania spp.

Objective. To identify the sand fly species of medically importance in the area of influence from Amoyá River Hydroelectric Project, Colombia.

Materials and methods. Sand flies were collected with CDC light traps, Shannon traps and sticky traps, from 15 villages in Chaparral County, Tolima.

Results. A total of 1,077 adult sand fly specimens were collected. Thirteen species were found in the genus Lutzomyia and one species in the genus Warileya. Among the Lutzomyia species, three species--Lutzomyia longiflocosa, Lutzomyia columbiana and Lutzomyia nuneztovari--are important for their epidemiological history. Lutzomyia suapiensis was a new record for Colombia, and Warileya rotundipennis was recorded for the first time in Tolima.

Conclusions. This study contributed to an increased knowledge of Colombian sand flies in terms of (1) expanding the geographical distribution of members of the subfamily Phlebotominae, (2) gaining estimates of species-richness and species associations in central Colombia, and (3) providing a better understanding of epidemiology of leishmaniasis in the Chaparral area.

Key words: Leishmaniasis, insect vectors, Lutzomyia, biodiversity, Andean ecosystem, Colombia

\section{Correspondencia:}

María Angélica Contreras, Programa de Estudio y Control de Enfermedades Tropicales, Universidad de Antioquia, Calle 62 №52-59, Medellín, Colombia

Teléfono: (574) 219 6502; fax: (574) 2196511

maria.contreras@pecet-colombia.org

Recibido: 29/08/11; aceptado:15/12/11
El conocimiento de la distribución geográfica y temporal de los flebotomíneos es necesario para el desarrollo de estrategias dirigidas a controlar estos vectores (1), reconocidos por su papel en la transmisión del parásito Leishmania (Ross, 1903), la bacteria Bartonella bacilliformis (Strong, et al., 1907) 
y algunos virus de los géneros Phlebovirus (Tesh, et al., 1976) y Vesiculovirus (Brown, et al., 1979) (2).

En la actualidad la riqueza de flebotomíneos en Colombia es relativamente grande y está representada por 162 especies: 152 de Lutzomyia (França, 1924), 8 de Brumptomyia (França y Parrot, 1924) y 2 de Warileya (Hertig, 1948), que se distribuyen en diversos nichos ecológicos y ecosistemas (3-5, Marín D, Ocampo C, Munstermann L, Ferro C. Actualización de la lista de flebotomíneos reportados para el departamento del Tolima, Colombia. Memorias, XXXV Congreso SOCOLEN, Cali 16, 17 y 18 de Julio. 2008; p. 135).

En el departamento de Tolima, Región Andina, se han llevado a cabo diversos estudios relacionados con la composición, biología de flebotomíneos e incriminación de vectores de leishmaniasis (6-8). Estos trabajos han documentado la presencia de 37 especies del género Lutzomyia ( $L u$.) distribuidas en seis grupos de especies, 11 subgéneros y una especie no agrupada. Lutzomyia longiflocosa (Osorno, Morales, Osorno y Muñoz, 1970) ha sido considerada como el vector más probable de leishmaniasis cutánea en el intradomicilio por su notable dominancia y aparente endofagia, en tanto que, Lu. columbiana (Ristorcelli y Van Ty, 1941) y Lu. nuneztovari (Ortiz, 1954) son consideradas vectores secundarios en el extradomicilio (8). En cuanto al agente etiológico, en el municipio de Chaparral, L. (Viannia) guyanensis (Floch, 1954) es el agente causal de la leishmaniasis cutánea en humanos y $L$. (V.) braziliensis (Vianna, 1911) y $L$. $(V$.) guyanensis en perros $(9,10)$.

Durante la ejecución del Programa de Prevención y Control de Enfermedades Tropicales del proyecto hidroeléctrico del río Amoyá, en el corregimiento Las Hermosas, municipio de Chaparral, Tolima, se desarrolló un estudio entomológico con el objetivo de evaluar la presencia de especies de flebotomíneos con importancia médica dentro del área de influencia.

\section{Materiales y métodos}

\section{Área de estudio}

Chaparral está localizado entre los 800 y los $4.500 \mathrm{msnm}\left(3^{\circ} 50^{\prime} \mathrm{N}\right.$ y $\left.75^{\circ} 34^{\prime} \mathrm{W}\right)$, al sur del departamento del Tolima sobre la cuenca alta del río Magdalena y se cataloga, según el sistema de clasificación de Holdridge (1977), como zona de bosque húmedo premontano (bh-PM), con un rango de precipitación de 1.500 a $2.000 \mathrm{~mm}$ y temperaturas medias anuales superiores a los
$24{ }^{\circ} \mathrm{C}$ (11). El municipio se localiza en la vertiente oriental de la Cordillera Central de los Andes colombianos y los muestreos se llevaron a cabo en 15 veredas correspondientes a Agua Bonita, Cimarrona Alta, San Jorge Bajo, San Roque, Maito, Cimarrona Baja, Porvenir, Angostura, El Moral, San Pablo Hermosas, Vegachiquita, La Virginia, Argentina Hermosas, Santa Bárbara y Río Negro. Estas áreas rurales ubicadas en el páramo de Las Hermosas, tienen como principales actividades económicas el cultivo de café, caña de azúcar y maíz, y la ganadería lechera.

\section{Muestreo e identificación del material entomológico}

La recolección de flebotomíneos fue realizada por tres investigadores del PECET durante cinco días por cada encuesta entomológica, entre junio de 2008 y mayo de 2009. Para el desarrollo de esta actividad se usaron 10 trampas de luz amarrilla de tipo CDC (entre las 18:00 y las 06:00) instaladas en el intradomicilio y el peridomicilio de las viviendas. También, se utilizaron trampas de tipo Shannon (entre las 18:00 y las 22:00); además, 1.196 trampas de papel impregnadas de aceite de ricino fueron ubicadas cada una con un área de $0,12 \mathrm{~m}^{2}$ $(21,6 \times 27,9 \mathrm{~cm})$, en 42 estaciones a lo largo de una intersección de 52 km.

El procesamiento del material recolectado incluyó la aclaración de los individuos en lactofenol (proporción 1:1) y el montaje sobre láminas portaobjeto con bálsamo de Canadá (12). La identificación de las especies de flebotomíneos se hizo mediante la morfología externa e interna comparada, siguiendo las claves de Young y Duncan (13) y Galati (14), además de las descripciones e ilustraciones de Torres-Espejo, et al., (15), Le Pont, et al., (16), Le Pont, et al., (17), Wolff y Galati (18), y Bejarano, et al. (19). Los especímenes están depositados en la "Colección de Vectores y Hospedadores Intermediarios de Enfermedades Tropicales" del Programa de Estudio y Control de Enfermedades Tropicales de la Universidad de Antioquia en Medellín, Colombia.

\section{Resultados}

Se obtuvieron 1.077 flebotomíneos, 692 (64,2\%) hembras y $385(35,8 \%)$ machos, de los cuales, el mayor porcentaje ( $n=752 ; 69,8 \%$ ) fue recolectado en trampas CDC, y en menor proporción en trampa Shannon ( $n=310 ; 28,9 \%)$ y adhesivas $(n=14 ; 1,3 \%)$.

Entre los ejemplares recolectados se encontraron 13 especies del género Lutzomyia y una especie del 
género Warileya (cuadro 1). Entre las especies del género Lutzomyia encontradas, Lu. longiflocosa, $L u$. columbiana y $L u$. nuneztovari sobresalen por sus historiales epidemiológicos. La especie dominante fue Lu. longiflocosa con 761 (70,8\%) individuos, distribuidos en el intradomicilio (12\%) y el peridomicilio (88\%). Se recolectó un menor número de individuos de Lu. columbiana ( $\mathrm{n}=89$; $8,3 \%)$, con mayor presencia en el peridomicilio $(88,8 \%)$ que en el intradomicilio $(11,2 \%)$. Con relación a Lu. nuneztovari, solo 10 ejemplares se recolectaron en el peridomicilio. Es notoria también la presencia de un considerable número de individuos ( $\mathrm{n}=128 ; 11,8 \%$ ) del subgénero Helcocyrtomyia (Barretto, 1962) (cuadro 1), cuyas especies se caracterizan por exhibir gran similitud en las formas de sus estructuras genitales.

Se resalta el hallazgo de un ejemplar de $L u$. suapiensis (Le Pont, Torrez-Espejo y Dujardin, 1997) (figura 1), que representa la primera notificación de esta especie para Colombia y de Warileya rotundipennis (Fairchild y Hertig, 1951) (figura 2), que es el primer representante de este género hallado en el departamento del Tolima.

A continuación se describen los patrones morfológicos de estas dos especies.

Lutzomyia suapiensis (Le Pont, Torrez-Espejo y Dujardin, 1997).

Material examinado. 1\%; Colombia, Tolima, Chaparral, vereda El Porvenir, $3^{\circ} 50^{\prime} 26,5^{\prime \prime} \mathrm{N}$, $75^{\circ} 34^{\prime} 29,0$ ", 3 de noviembre de 2008. Trampa CDC (Colección de Vectores y Hospedadores Intermediarios de Enfermedades Tropicales).

Las características morfológicas observadas en el ejemplar recolectado se describen a continuación: cabeza, cibario con cuatro dientes horizontales, equidistantes; ascoides simples sobre el flagelómero II, sin sobrepasar el ápice del segmento, quinto palpómero más largo que el tercer palpómero; genitales: el individuo de Lu. suapiensis capturado en Tolima (figura 1), presenta la espermateca característica de las hembras de la serie pia (Galati, 1995), estriada en forma de saco con un anillo liso apical con bordes rectos. El conducto común es más largo, casi el doble, que los conductos individuales. La pleura está pigmentada basalmente. La longitud del labroepifaringe es de $293 \mu \mathrm{m}$ y la del segundo palpómero es igual a $148 \mu \mathrm{m}$, lo que corresponde con los caracteres diagnósticos propuestos por Le Pont, et al., para Lu. suapiensis.

Warileya rotundipennis (Fairchild y Hertig, 1951)

Material examinado. 2 , Colombia, Tolima, Chaparral, vereda Angostura, $3^{\circ} 47^{\prime} 38,5^{\prime \prime} \mathrm{N}, 75^{\circ}$ 34' 16,1" W, 1120 m, 1 de junio de 2008, trampa CDC (Colección de Vectores y Hospedadores Intermediarios de Enfermedades Tropicales).

Las hembras de $W$. rotundipennis recolectadas se caracterizan por presentar sutura interocular completa, distancia interocular equivalente a la mitad del ancho del ojo, clípeo con cerdas,

Cuadro 1. Número de individuos recolectados por especie de flebotomíneo y por tipo de trampa en la zona de influencia del proyecto hidroeléctrico Río Amoyá, (Chaparral, Tolima)

\begin{tabular}{|c|c|c|c|c|c|c|c|c|c|c|}
\hline \multirow{3}{*}{ Especie } & \multicolumn{6}{|c|}{ Tipo de trampa } & \multirow{2}{*}{\multicolumn{2}{|c|}{ Total }} & & \\
\hline & \multicolumn{2}{|c|}{ CDC } & \multicolumn{2}{|c|}{ Adhesiva } & \multicolumn{2}{|c|}{ Shannon } & & & \multirow{2}{*}{\multicolumn{2}{|c|}{$q+\mathbf{N}(\%)$}} \\
\hline & $q \mathbf{n}$ & on & $q \mathbf{n}$ & $n$ & $q \mathbf{n}$ & $3 n$ & $q_{N}$ & $\mathbf{N}$ & & \\
\hline 1. Lu. longiflocosa* & 349 & 236 & 7 & 3 & 114 & 72 & 470 & 311 & 781 & $(72,5)$ \\
\hline 2. Lu. columbiana* & 68 & 15 & - & 1 & 5 & - & 73 & 16 & 89 & $(8,3)$ \\
\hline 3. Lu. trinidadensis & 5 & - & 1 & - & - & 9 & 6 & 9 & 15 & $(1,4)$ \\
\hline 4. Lu. erwindonaldoi & 13 & - & - & - & 35 & 2 & 48 & 2 & 50 & $(4,6)$ \\
\hline 5. Lu. carpenteri & 8 & 5 & - & - & - & - & 8 & 5 & 13 & $(1,2)$ \\
\hline 6. Lu. nuneztovari * & 1 & 3 & - & - & 5 & 1 & 6 & 4 & 10 & $(0,9)$ \\
\hline 7. Lu. shannoni * & 1 & 1 & - & - & 1 & 3 & 2 & 4 & 6 & $(0,6)$ \\
\hline 8. Lu. lerayi & - & 2 & - & - & - & 1 & - & 3 & 3 & $(0,3)$ \\
\hline 9. Lu. lichyi & - & - & - & 2 & - & - & - & 2 & 2 & $(0,2)$ \\
\hline 10. Lu. suapiensis ${ }^{++}$ & 1 & - & - & - & - & - & 1 & - & 1 & $(0,1)$ \\
\hline 11. Lu. sordellii & 1 & - & - & - & - & - & 1 & - & 1 & $(0,1)$ \\
\hline 12. Lu. (Helcocyrtomyia) sp. & 18 & - & - & - & 36 & 24 & 54 & 24 & 78 & $(7,2)$ \\
\hline 13. Lutzomyia sp. & 18 & 5 & - & - & 3 & - & 21 & 5 & 26 & $(2,4)$ \\
\hline 14. W. rotundipennis ${ }^{+}$ & 2 & - & - & - & - & - & 2 & - & 2 & $(0,2)$ \\
\hline Total & 485 & 267 & 8 & 6 & 199 & 112 & 692 & 385 & 1.077 & $(100)$ \\
\hline
\end{tabular}

†: hembra; ô: macho; n: número de individuos; N: número total de individuos; *: flebotominos de importancia médica; +: nuevo registro para el departamento de Tolima; ${ }^{++}$: nuevo registro para Colombia. 

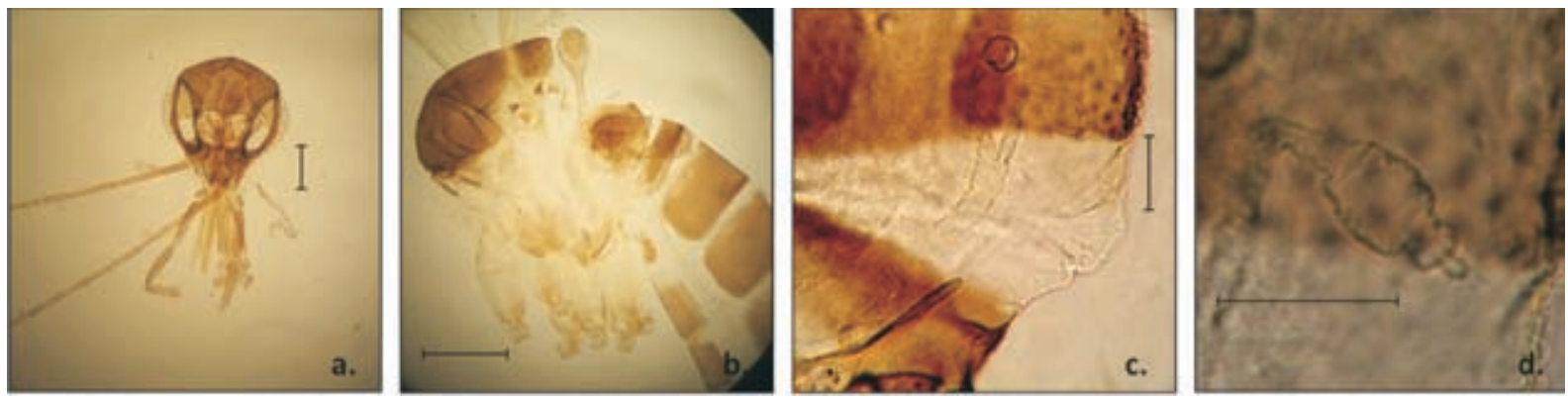

Figura 1. Lutzomyia suapiensis (Le Pont, Torrez-Espejo \& Dujardin, 1997) 9 a) cabeza, barra=117 $\mu \mathrm{m}$; b) tórax, barra= $341 \mu \mathrm{m}$; c) espermatecas, conductos individuales y común, barra $=48 \mu \mathrm{m}$; d) cabeza de la espermateca, barra=44 $\mu \mathrm{m}$
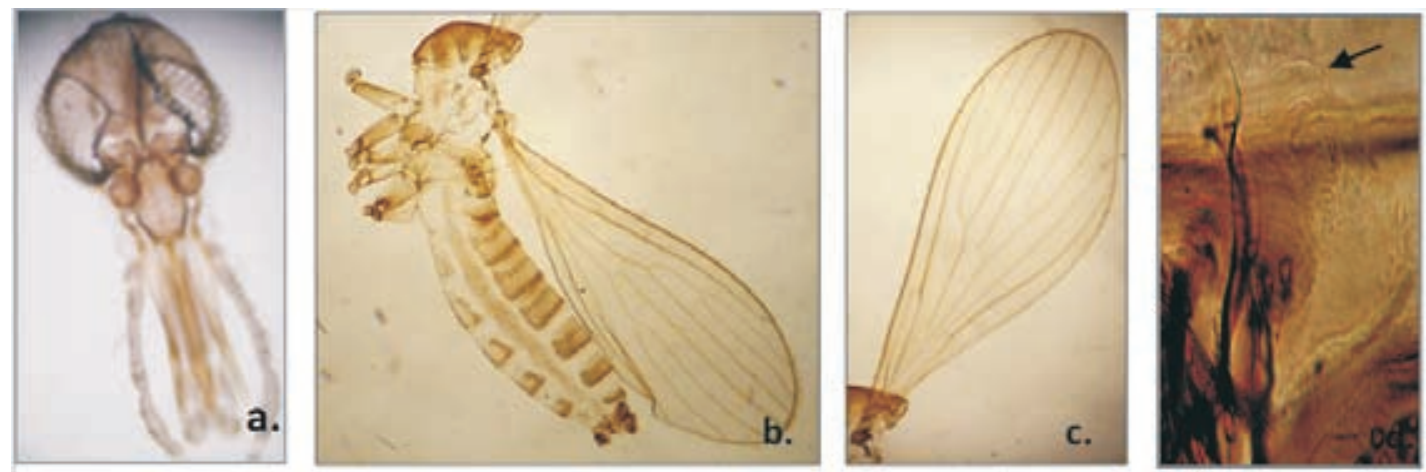

Figura 2. Warileya rotundipennis (Fairchild y Herting, 1951) ㅇ a) cabeza; b) tórax, ala y abdomen; c) ala; d) espermatecas y furca

longitud del quinto palpómero equivalente a la de segundo palpómero y ausencia de armadura bucal (figura 2a). El tórax está desprovisto de cerdas proepimerales y anepisternales superiores (figura $2 b)$. En el abdomen, los tergitos se observan con cerdas dispuestas en dos líneas transversales (figura 2b). Las espermatecas presentan cuerpo tubular anillado y cabeza en forma de botón esférico (figura 2d).

\section{Discusión}

Entre las especies de importancia epidemiológica en el área de influencia del proyecto hidroeléctrico del río Amoyá, se destaca Lu. longiflocosa por su abundancia en el peridomicilio, lo que evidencia un posible comportamiento endofágico y su potencial papel como vector, por sus antecedentes históricos en la trasmisión de leishmaniasis cutánea originada por L. (V.) braziliensis en algunas localidades de los departamentos del Tolima, Huila y Norte de Santander (9,20, Pardo R, Ferro C, Lozano G, Lozano C, Cabrera O, Davies C. Flebótomos (Diptera: Psychodidae) vectores de leishmaniasis cutánea y sus determinantes ecológicos en la zona cafetera del departamento del Huila. Memorias, XXVI Congreso de la Sociedad Colombiana de Entomología, Bogotá, 1999; p. 147).
Los otros hallazgos interesantes en el panorama de la transmisión de la leishmaniasis, corresponden a Lu. columbiana y Lu. nuneztovari, especies que también se han asociado en Colombia con la transmisión de $L$. (V.) braziliensis y L. (Leishmania) mexicana (Biagi, 1953) (21-24). Estas tres especies pertenecen al grupo verrucarum, reconocido por incluir varias especies endémicas de la zona montañosa de la Región Andina, cuya preferencia de hábitats está asociada con los ecosistemas cafeteros $(19,21)$.

El presente informe constituye la primera notificación del taxón. Lu. suapiensis para Colombia. Con respecto a la taxonomía, y según el esquema de clasificación de Galati (14), esta especie es incluida en la serie pia del subgénero Pifanomyia (Ortiz y Scorza, 1963) del género Pintomyia (Costa Lima, 1932), junto a otras ocho especies, que corresponden a: Lu. pia (Fairchild y Hertig, 1961); Lu. torrealbai (Martins, Fernandez y Falcão, 1979); Lu. valderramai (Cazorla, 1988); Lu. reclusa (Fernandezy Rogers, 1991); Lu. tihuiliensis(LePont, Torrez-Espejo y Dujardin,1997); Lu. tocaniensis (Le Pont, Torrez-Espejo y Dujardin,1997); Lu. limafalcaoae (Wolff y Galati, 2002), y Lu. emberai (Bejarano, Duque y Vélez, 2004). 
Se ha reconocido a Lu. suapiensis por su comportamiento antropofílico en áreas de Bolivia y Perú, donde la leishmaniasis es endémica $(16,25)$. Aunque se desconocen las preferencias ecológicas de esta especie, Lu. suapiensis, Lu. tihuiliensis y Lu. tocaniensis se han encontrado en simpatría en Bolivia y Perú $(17,25)$. Hasta la fecha, solo cuatro especies de la serie pia están registradas en Colombia: Lu. pia, Lu. limafalcaoae, $L u$. emberai y $L u$. tihuiliensis. Dentro de la serie pia, Lu. suapiensis se encuentra estrechamente relacionada con. Lu. tihuiliensis, pero se diferencia por caracteres morfométricos cefálicos correspondientes al labroepifaringe y el segundo palpómero $(14,16,18)$.

Otro aporte significativo a la riqueza de flebotomíneos en el departamento del Tolima, lo constituye el género Warileya, el cual cuenta con siete especies descritas, que se encuentran distribuidas desde Costa Rica hasta Bolivia (13). Para Colombia se han informado dos especies, $W$. nigrosacculus (Fairchild y Hertig, 1951) y W. rotundipennis (3). La última se ha registrado en los departamentos de Antioquia, Chocó, Norte de Santander, Risaralda y Valle del Cauca (3), por lo cual éste constituye el primer informe para el departamento del Tolima. Por su morfología, las dos especies se diferencian entre sí por el cuerpo de la espermateca, el cual exhibe una forma vesiculosa en $W$. nigrosacculus y una tubular en $W$. rotundipennis (figura 2d).

El hallazgo de individuos del género Warileya es un posible indicio de la preexistencia de coberturas vegetales, ya que los psicódidos se han contemplado como indicadores de la calidad de hábitat en bosques tropicales $(7,26)$, pero la baja densidad de especímenes recolectados indica que el área ha sido perturbada por actividades relacionadas con la extensión de las prácticas agronómicas y los crecientes procesos de urbanización no planificados.

En conclusión, el presente trabajo permitió registrar por primera vez en Colombia, la presencia de la especie Lu. suapiensis y notificar para el departamento del Tolima a Warileya rotundipennis. Estos hallazgos contribuyen al conocimiento de la distribución geográfica de la subfamilia Phlebotominae en el país, lo que favorece la comprensión de la biodiversidad, taxonomía y sistemática de estos insectos, aspectos fundamentales para la incriminación vectorial y para establecer sus relaciones ecológicas en áreas con transmisión de leishmaniasis.

\section{Agradecimientos}

A las distintas comunidades del municipio Chaparral por su colaboración, a la Unidad de Ecoepidemiología (PECET, Universidad de Antioquia) por la recolección de los insectos y a Isagén.

\section{Conflicto de intereses}

Los autores declaran que no existen conflictos de intereses.

\section{Financiación}

Este estudio recibió el apoyo financiero de Isagén (contrato $N^{\circ} 46 / 2708$ ) y del Programa de Estudio y Control de Enfermedades Tropicales, PECET, de la Universidad de Antioquia.

\section{Referencias}

1. Ibáñez B, Rodríguez C, Gómez H, Esquinca J. First record of Lutzomyia evansi (Nuñez-Tovar 1924) in México (Diptera: Psychodidae, Phlebotominae). Mem Inst Oswaldo Cruz. 2004;99:127-9.

2. Acevedo M, Arrivillaga J. Eco-epidemiología de flebovirus (Bunyaviridae, Phlebovirus) transmitidos por flebótomos (Psychodidae, Phlebotominae). Bol Malar Salud Ambient. 2008;48:3-16.

3. Bejarano E. Lista actualizada de los psicódidos (Diptera: Psychodidae) de Colombia. Folia Entomol Mex. 2006;45: 47-56.

4. Cabrera OL, Mosquera L, Santamaría E, Ferro C. Flebótomos (Diptera: Psychodidae) del departamento de Guaviare, Colombia con nuevos registros para el país. Biomédica. 2009;29:73-86.

5. Bejarano E, Vivero R, Uribe S. Description of Lutzomyia velezi, a new species of phlebotomine sand fly (Diptera: Psychodidae) from the Department of Antioquia, Colombia. Mem Inst Oswaldo Cruz. 2010;105:322-5.

6. Alexander B, Agudelo L, Navarro F, Ruiz F, Molina J, Aguilera G, et al. Phlebotomine sandflies and leishmaniasis risks in Colombian coffee plantations under two systems of cultivation. Med Vet Entomol. 2001;15:364-73.

7. Morales D, Castaño C, Lozano E, Vallejo H. Descripción de la epidemia de leishmaniasis cutánea en Chaparral y San Antonio, Tolima, 2003 y 2004 (semana 24). Inf Quin Epidemiol Nac. 2004;9:180.

8. Pardo R, Cabrera O, Becerra J, Fuya P, Ferro C. Lutzomyia longiflocosa, posible vector en un foco de leishmaniasis cutánea en la región subandina del departamento del Tolima, Colombia, y el conocimiento que tiene la población sobre este insecto. Biomédica. 2006;26:95-108.

9. Rodríguez B, Góngora I, Prager R, Pacheco M, Montero $\mathbf{R}$, Navas L, et al. Etiologic agent of an epidemic of cutaneous leishmaniasis in Tolima, Colombia. Am J Trop Med Hyg. 2008;78:276-82.

10. Santaella J, Ocampo C, Saravia N, Méndez F, Góngora $\mathbf{R}$, Gómez A, et al. Leishmania (Viannia) infection in the 
domestic dog in Chaparral, Colombia. Am J Trop Med Hyg 2011;84:674-80.

11. Instituto Geográfico Agustín Codazzi, IGAC. Diccionario Geográfico de Colombia. Tercera edición. Santafé de Bogotá: Instituto Geográfico Agustín Codazzi; Santa Fe de Bogotá: Reprolaser Ltda.; 1996. p. 2459-60.

12. Maroli M, Feliciangeli D, Arias J. Métodos de captura, conservación y montaje de los flebotomos (Diptera: Psychodidae). Washington D.C.: OPS/OMS/HCP/JCT/ 95/97; 1997. p. $1-72$.

13. Young D, Duncan M. Guide to the identification and geographic distribution of Lutzomyia sand flies in Mexico, the West Indies, Central and South America (Diptera: Psychodidae). Mem Am Entomol Inst. 1994;54:1-881.

14. Galati E. Phlebotominae (Diptera, Psychodidae). Classificação morfologia e terminologia e identificação de adultos. São Paulo: Universidade de São Paulo, Apostila disciplina HEP 5752: Bioecologia e identificação de Phlebotominae; 2010.

15. Torres J, Cáceres G, Pont L. Description de deux nouvelles espéces de Phlebotomes du Sous-Genre Helcocyrtomyia, du piémont andin bolivien (Diptera, Psychodidae). Parasite. 1995;2:157-62.

16. Le Pont, Torres E, Dujardin J. Phlébotomes de Bolivie: Description de quater nouvelles espéces de Lutzomyia (Diptera: Psychodidade). Ann Soc Entomol Fr. 1997;33:5564.

17. Le Pont F, Martínez E, Torres-Espejo E, Dujardin J. Phlébotomes de Bolivie: description de cinq nouvelles espèces de Lutzomyia de la région subandine (Diptera, Psychodidae). Bull Soc Entomol Fr. 1998;103:159-73.

18. Wolff M, Galati E. Description of Pintomyia limafalcaoae and Pintomyia antioquiensis, two new species of phlebotomine sandfly (Diptera, Psychodidae) from the Colombian Andes. Mem Inst Oswaldo Cruz. 2002;97:317-24.
19. Bejarano E, Duque P, Vélez I. Taxonomy and distribution of the series pia of the Lutzomyia verrucarum group (Diptera: Psychodidae), with a description of Lutzomyia emberai $\mathrm{n}$. sp. J Med Entomol. 2004;4:833-41.

20. Cárdenas R, Pabón E, Anaya H, Sandoval C. Presencia de Lutzomyia longiflocosa (Diptera: Psychodidae) en el foco de leishmaniasis tegumentaria americana del municipio de Ábrego, Norte de Santander. Primer registro para el departamento. Clon. 2005;3:7-14.

21. Montoya J, Jaramillo J, Palma G, Gómez T, Segura I, Travi B. Report of an epidemic outbreak of tegumentary leishmaniasis in a coffee-growing area of Colombia. Mem Inst Oswaldo Cruz. 1990;85:119-21.

22. Alexander B, Ferro C, Young C, Morales A, Tesh R. Ecology of Phlebotomine sand flies (Diptera: Psychodidae) in a focus of Leishmania (Viannia) braziliensis in a northeastern Colombia. Mem Inst Oswaldo Cruz. 1992;87:387-95.

23. Montoya-Lerma J, Ferro C. Flebótomos (Diptera: Psychodidae) de Colombia. En: Amat G, Andrade MG, Fernández $F$, editores. Insectos de Colombia. Colección Jorge Álvarez Lleras No. 13. Bogotá: Academia Colombiana de Ciencias Exactas, Físicas y Naturales; 1999. p. 211-45.

24. Santamaría E, Castillo M, Cárdenas R, Bello F, Ayala M, Ferro C. Competencia vectorial de las especies de Lutzomyia del grupo verrucarum (Diptera, Psychodidae) en un foco endémico de Leishmania braziliensis en Reventones, Cundinamarca. Biomédica. 1999;19:115-26.

25. Cáceres G, Quate L, Galati E, Baht H. Flebotominos (Diptera: Psychodidae) de San Pedro, distrito Kosñipata, Paucartambo - Cusco, y nuevos reportes para el Perú. Rev Peru Med Exp Salud Pública. 2001;18:24-6.

26. Barrett T, Freitas R, Albuquerque C, Hurtado J. Report on a collection of phlebotomine sand flies (Diptera: Psychodidae) from the middle Solimões (Amazonas, Brazil). Mem Inst Oswaldo Cruz. 1996;91:27-35. 